\title{
Buccal venom gland associates with increased of diversification rate in the fang blenny fish Meiacanthus (Blenniidae; Teleostei)
}

\author{
Shang-Yin Vanson Liu ${ }^{\mathrm{a}, *}$, Bruno Frédérich ${ }^{\mathrm{b}, 1}$, Sébastien Lavouéc, Jonathan Chang ${ }^{\mathrm{d}}$, \\ Mark V. Erdmann ${ }^{\mathrm{e}}$, Gusti Ngurah Mahardika ${ }^{\mathrm{f}}$, Paul H. Barber ${ }^{\mathrm{d}}$ \\ a Department of Marine Biotechnology and Resources, National Sun Yat-Sen University, Kaohsiung 80424, Taiwan \\ ${ }^{\mathrm{b}}$ Laboratoire d'Océanologie, UR FOCUS, Université de Liège, 4000 Liège, Belgium \\ ${ }^{\mathrm{c}}$ Institute of Oceanography, National Taiwan University, Roosevelt Road, Taipei 10617, Taiwan \\ ${ }^{\mathrm{d}}$ Department of Ecology and Evolutionary Biology, University of California Los Angeles, Los Angeles, CA 90095-7239, USA \\ e Conservation International Indonesia Marine Program, 80235 Bali, Indonesia \\ ${ }^{\mathrm{f}}$ The Indonesian Biodiversity Research Centre, The Animal Biomedical and Molecular Biology Laboratory of Udayana University, Jl Sesetan-Markisa 6, Denpasar, Bali, \\ Indonesia
}

\section{A R T I C L E I N F O}

\section{Keywords:}

Macroevolution

Innovation

Diversification rate shift

Coral reef fishes

\begin{abstract}
A B S T R A C T
At the macroevolutionary level, many mechanisms have been proposed to explain explosive species diversification. Among them morphological and/or physiological novelty is considered to have a great impact on the tempo and the mode of diversification. Meiacanthus is a genus of Blenniidae possessing a unique buccal venom gland at the base of an elongated canine tooth. This unusual trait has been hypothesized to aid escape from predation and thus potentially play an important role in their pattern of diversification. Here, we produce the first time-calibrated phylogeny of Blenniidae and we test the impact of two morphological novelties on their diversification, i.e. the presence of swim bladder and buccal venom gland, using various comparative methods. We found an increase in the tempo of lineage diversification at the root of the Meiacanthus clade, associated with the evolution of the buccal venom gland, but not the swim bladder. Neither morphological novelty was associated with the pattern of size disparification in blennies. Our results support the hypothesis that the buccal venom gland has contributed to the explosive diversification of Meiacanthus, but further analyses are needed to fully understand the factors sustaining this burst of speciation.
\end{abstract}

\section{Introduction}

Understanding the evolutionary causes underlying species diversification is a crucial task for evolutionary biologists. External factors such as habitat shifts have been shown to have a profound effect on the net diversification rate and/or the rate of phenotypic evolution in several marine fish groups (Alfaro et al., 2007; Betancur et al., 2012; Frédérich et al., 2016; Price et al., 2013; Santini et al., 2013; Sorenson et al., 2014a, 2014b). Morphological or physiological innovations are internal factors thought to be strong evolutionary driving forces of diversification and disparification (e.g. Carlson et al., 2011; Frédérich et al., 2014; Helmstetter et al., 2016; Near et al., 2012). Within the framework of adaptive radiation (Simpson, 1953), a novelty may be a "key innovation" when evidence supports its positive effect on subsequent lineage and phenotypic diversification (Losos, 2010). However an increase of diversification rates following a change in morphological or physiological traits is not guaranteed due to complex interactions between the positive effect on performance, ecological opportunity and functional trade-offs (Levinton, 1988). As what have been illustrated in recent macroevolutionary studies (e.g. Alfaro et al., 2009; Near et al., 2012), a burst of diversification expected under the "key innovation" hypothesis (Heard and Hauser, 1995) may never happen or it may occur later when ecological opportunities are created (e.g. loss of competitor, changes in environmental conditions). Additionally, innovations may be not associated with subsequent ecological diversification but may lead to ecological specialization, i.e. a new adaptive peak on the adaptive landscape (Frédérich et al., 2014; Olivier et al., 2017; Wainwright and Price, 2016).

The production of venom has long been suggested as being responsible for the diversification of extant snakes (Vidal, 2002) and lizards (Fry et al., 2006). Among ray-finned fishes, those producing venom can be found in catfishes (Siluriformes), toadfishes

\footnotetext{
* Corresponding author.

E-mail address: oceandiver6426@gmail.com (S.-Y.V. Liu).

${ }^{1}$ Contribute equally as 1 st author.
} 
(batrachoidids), scorpionfishes (Scorpaenidae), surgeonfishes (Acanthuroidei), scats (Scatophagidae), rabbitfishes (Siganidae), sabertoothed blennies (Blennioidei), jacks (Carangidae), stargazers (Uranoscopidae), and weeverfishes (Trachinidae) (Smith and Wheeler, 2006). Function of these venom organs in most of these fishes is clearly defense as they are present in locations such as spines, cleithrum, and opercle, where envenomation can only occur if the fish is grasped or bitten. Only the species in the genus Meiacanthus (Blenniidae, Nemophini), also known as fang blennies, have a venomous organ specialized as a buccal venom gland at the base of the canine tooth with a deep groove along the anterior surface. It is postulated that this proximity of tooth and gland helps inject venom secreted by the gland (Fishelson, 1974). Recently, it has been documented that their venom contains a number of toxic components that can result in a multifunctional biochemical phenotype that exerts potent hypotensive, weakly neurotoxic, and proinflammatory bioactivity in rats (Casewell et al., 2017). While there is no conclusive evidence of the function of this buccal venom gland, field observations and preliminary experiments suggest that Meiacanthus can attack from the inside of a predator's mouth and then escape (Smith-Vaniz et al., 2001). The benefit of protection from predators under the symbiotic relationship between clownfishes and anemones has been suggested contributing to the diversification of $\mathrm{Am}$ phiprion in the Pomacentridae (Litsios et al., 2012). Although there is no conclusive evidence of the function of the buccal venom gland, it is possible that the presence of the unique venom gland defensive mechanism similarly stimulates diversification in Meiacanthus.

The tribe Nemophini within Blenniidae comprises six genera, including Adelotremus, Xiphasia, Meiacanthus, Aspidontus, Plagiotremus, and Petroscirtes (Smith-Vaniz, 1976, 2017)]. Except for two species of Xiphasia and two species of Adelotremus, Nemophini have a well-developed swim bladder that allows them to actively forage in the water column. Unlike most of the benthic blennies, which typically lack swim bladder and feed mainly on algae and detritus, Nemophini species are carnivores and omnivores (Shaun, 2009). Aspidontus and Plagiotremus are fin/scale feeders, while Meiacanthus species actively search for small benthic invertebrates and zooplankton in the water column (Smith-Vaniz, 1976, 1987). Their semi-pelagic life, supported by the presence of a swim bladder, seems to provide an opportunity for exploring untapped niches and possibly facilitating lineage and phenotypic diversification in the Nemophini.

In this study, we examined whether a shift in the diversification rate occur in the lineages of Blenniidae that possess either buccal venom gland or swim bladder. Specifically, we tested whether the presence of the buccal venom gland and swim bladder results in an increase of evolutionary diversification rates in comparison to other blennies. Our results suggest that, contrary to the presence of swim bladder, the buccal venom gland is associated with a variation in the rate of lineage diversification in Meiacanthus. On the other hand, we found no evidence of an increase of the rate of size evolution following these innovations.

\section{Materials and methods}

\subsection{Fish specimen collection and data acquisition}

We obtained Meiacanthus specimens through museum collections, purchases of wild-caught individuals via the aquarium trade, and collections of live samples in the field using scuba equipment. Species were identified based on diagnostic morphological traits described in the literature (Allen and Erdmann, 2012; Smith-Vaniz, 1976, 1987). In total, we sampled 15 Meiacanthus species representing $60 \%$ of generic diversity based on the latest taxonomic review (Hastings and Springer, 2009). For systematic comparison, we included species from the tribe Nemophini, to which Meiacanthus belongs, as well as representatives of 13 unranked monophyletic clades including Blenniidae, Ecsenius, Springerichthys, Nemophini, Plagiotrematinae, Smithvanizichthys, Omobranchus, Almadablennius, Blenniini, Salariini, Salarias, and
Williamsichthys used in a recently published Blenniidae phylogeny (Hundt et al., 2014). In total, our taxonomic sampling included 19 species with novel sequences and sequences of 104 species acquired from GenBank along with one outgroup (Supplementary Table S1).

Maximum body size (total length) was used as an ecomorphological trait related to niche partitioning in blennies. We gathered morphological data (i.e. venom gland, swim bladder and body size) from various sources, including FishBase and the primary literature (Hastings and Springer, 2009; Smith-Vaniz, 1976, 1987). For every species in our study, we scored two binary variables: presence (1)/absence (0) of buccal venom gland, and presence (1)/absence (0) of swim bladder.

Total genomic DNA was extracted from muscle or fin clips using Qiagen ${ }^{\circledR}$ DNAeasy Blood and Tissue Kit (Qiagen, Valencia, CA) following the manufacturer's protocol. To combine our data with a recently published study (Hundt et al., 2014), the same four nuclear exons were amplified: ENC1, myh6, ptr, and tbr1. These molecular markers are well-conserved, single-copy nuclear genes that are phylogenetically informative ( $\mathrm{Li}$ et al., 2007). PCR reactions contained $1.5 \mu \mathrm{l}$ template DNA, $2.75 \mu$ l water, $6.25 \mu$ l GoTaq ${ }^{\circledR}$ Green Master Mix (Promega, Madison, WI), $1.0 \mu \mathrm{l}$ forward primer, and $1.0 \mu \mathrm{l}$ reverse primer. Forward and reverse primer designs and PCR cycling followed original protocols (Li et al., 2007). The purification of PCR products and sequencing reactions were done by Genomics BioSci and Tech. Nucleotide sequences of forward and reverse strands were determined using an ABI 3730XL automated sequencer (Applied Biosystems, Carlsbad, California). Sequences were assembled and edited using Sequencher version 4.2 software (Gene Codes, Ann Arbor, Michigan).

\subsection{Phylogenetic reconstruction, divergence time estimation and stochastic mapping}

We jointly reconstructed the phylogenetic relationships and divergence times of Meiacanthus within the Blenniidae using a partitioned Bayesian method as implemented by BEAST v.1.8.2 (Drummond and Rambaut, 2007). We concatenated all four markers into one data matrix and determined the best fitting model of sequence evolution for each locus using jModelTest2 (Darriba et al., 2012). Within the concatenated dataset, each of the four loci was allowed to have its own evolutionary rate under a GTR $+\mathrm{G}$ model of substitutions. We unlinked substitution models, rate heterogeneity models, and base frequencies across partitions. We built the BEAST input XML file using BEAUti v.1.8.2.

Using BEAST, we conducted two separate runs of $5 \times 10^{7}$ generations each with trees and values sampled once every 1000 generations. Each run was checked for convergence with Tracer v1.6 (http://tree. bio.ed.ac.uk/software/tracer/). After removing the burn-in portion of each run $(20 \%)$, the remaining tree samples from the two runs were pooled into a combined file and maximum clade credibility tree with posterior mean divergence ages and $95 \%$ credibility intervals (CI) were assigned to nodes using TreeAnnotator v1.8.2 (Drummond and Rambaut, 2007).

Blenniid fossils are relatively rare, and often very fragmented (Bannikov, 1998) and not informative enough to provide reliable calibration points. The oldest known blenniid fossil is Oncolepis isseli from the Eocene deposits of Monte Bolca (Italy). However, the phylogenetic position and assignment of this fossil as a blenniid was recently reevaluated as uncertain (Bannikov, 2014). Tottoriblennius hiraoi is a fossil having some morphological affinities to the tribe Nemophini (Yabumoto and Uyeno, 2007). However, it is a young fossil, dated to only mid-Miocene. Therefore, to time-calibrate our tree, we use a secondary calibration from other, more taxonomically inclusive studies. A recent large-scale phylogeny of spiny-rayed fishes time-calibrated with several fossils offered the most comprehensive phylogenetic timetree of the Acanthomorpha (Near et al., 2013), and estimated the crown Blenniidae to have evolved roughly 60-65 million years ago (Mya). Following this, we constrained the age of crown Blenniidae in our tree to about 66 Mya, corresponding to the Cretaceous-Paleogene limit (K- 
Pg boundary). A detail explanation on fossil and secondary calibration point selection is given in the supplementary information.

We used stochastic character mapping to infer possible morphological histories. This mapping was produced using the function make.simmap in the R package phytools (version 0.5.38) (Revell, 2012). For the parameterization of make.simmap, we used the estimated ancestral state of each morphological traits (presence/absence of the venom gland or swim bladder) and the best model for the transition matrix from our empirical data; i.e., a model with equal rates of transition (See BiSSE results).

\subsection{Dynamics of lineage diversification}

In order to detect changes in the diversification dynamics (i.e. speciation and extinction rates) on the dated phylogenetic tree, we used BAMM 2.5 (Rabosky et al., 2014), which accounts for missing data in a given phylogeny to detect the rate shift on the dated phylogenetic tree of Blenniidae. All settings used in the analyses are provided in the BAMM control files (Supplementary material). The sampling fraction of each genus (Supplementary material) was determined by comparing the number of taxa sampled with the total number of blennies (Hastings and Springer, 2009). Instead of finding a single best-fit configuration of rate shifts, BAMM simulates posterior distributions of rate-shift configurations. Thus, it accounts for incomplete taxon sampling analytically and uses reversible-jump Markov chain Monte Carlo (MCMC) computation to select among models that vary in the number of diversification regimes, thereby accounting for rate variation through time and among lineages. We ran two MCMCs for $10^{7}$ generations with a sampling frequency of 1000 for each clade. Using the BAMMtools package (Rabosky et al., 2014) in R 2.15.1 (R Core Team, 2013), we checked for convergence by plotting the log-likelihood trace of the MCMC output file and estimated the effective population size by using tools from the coda package (Plummer et al., 2006).

We used several approaches to detect possible rate shifts, including a $95 \%$ credible set of distinct shift configurations. We also calculated marginal shift probabilities and cumulative shift probabilities to support the rate shift configurations observed. To test the sensitivity of the expected number of prior rate shifts, we set the expected prior shifts to 0.1 (compared to a default of 1.0) and used a Bayes factor criterion (Shi and Rabosky, 2015) to test a model with no rate shifts against a model with one rate shift. To visualize the complex rate shift dynamics on the tree, we applied macroevolutionary cohort analysis. These shifts in diversification dynamics are referred to as initiation radiations, except where there is a slowdown in net diversification rate. We summarized BAMM analyses by computing time-specific rates of clade-specific rates. Finally, to estimate clade-specific rates of lineage diversification (Meiacanthus only vs. without Meiacanthus), we computed the mean rate over all branches assigned to a given clade, weighted by the length of the branch.

In addition to BAMM analyses, we used the BiSSE (Maddison et al., 2007) method to test whether both innovations, i.e. swimbladder and venom gland, induced variation in the rates of lineage diversification. Briefly, BiSSE (Maddison et al., 2007) uses a character-dependent diversification model, and tests whether the presence or absence of a character ( 0 or 1$)$ is associated with different speciation $\left(\lambda_{0}\right.$ and $\left.\lambda_{1}\right)$ or extinction $\left(\mu_{0}\right.$ and $\left.\mu_{1}\right)$ rates, and estimates the rate of transition between states $\left(\mathrm{q}_{01}\right.$ and $\left.\mathrm{q}_{10}\right)$. BiSSE analyses were performed in the R-package diversitree 0.9-3 (FitzJohn, 2012). For both morphological characters, four models were parameterized over 1000 randomly sampled trees from the post burn-in sample: (1) a full BiSSE model, where speciation, extinction, and transition rates may vary independently $\left(\lambda_{0} \neq \lambda_{1}\right.$, $\mu_{0} \neq \mu_{1}, \mathrm{q}_{01} \neq \mathrm{q}_{10}$ ), (2) an equal speciation model where $\lambda$ are constrained $\left(\lambda_{0}=\lambda_{1}\right)$, (3) an equal extinction model where $\mu$ and $q$ are constrained $\left(\mu_{0}=\mu_{1}, q_{01}=q_{10}\right)$, and (4) the BiSSE null model where $\lambda$, $\mu$, and $\mathrm{q}$ are constrained $\left(\lambda_{0}=\lambda_{1}, \mu_{0}=\mu_{1}, q_{01}=q_{10}\right)$.

To avoid high support for a BiSSE model (Rabosky and Goldberg,
2015) where an unobserved, hidden trait could also contribute to a difference in diversification rates across a phylogeny, we also tested a two-state character-independent diversification model as implemented in the R package hisse (Beaulieu and O'Meara, 2016). This hidden trait, with states labeled as A and B, could account for an increase in diversification rate separate from the presence or absence of the observed trait, in this case the buccal venom gland. We therefore parameterized three character independent models: (1) an all rates different model, where diversification rates were permitted to vary with respect to a hidden character, and all transition rates were permitted to be unequal (e.g., $\lambda_{0 \mathrm{~A}} \neq \lambda_{0 \mathrm{~B}}, \lambda_{1 \mathrm{~A}} \neq \lambda_{1 \mathrm{~B}}, \lambda_{0 \mathrm{~A}}=\lambda_{1 \mathrm{~A}}, \lambda_{0 \mathrm{~B}}=\lambda_{1 \mathrm{~B}}$ ); (2) a symmetric transition rates model, with diversification rates as in model 1 (e.g., $\mathrm{q}_{\mathrm{OA}->}>$ ОВ $\left.=\mathrm{q}_{\mathrm{OB}-}>\mathrm{OA}\right)$, (3) and an equal transition rates model, where all transition rates are constrained to be the same. We compared these three character-independent diversification models to the full BiSSE and BiSSE null models in an AIC framework.

\subsection{Pattern of size disparification}

To test whether morphological innovations induced variation in the pattern of size disparification throughout the evolution of blennies, we fitted six different models: (1) a single-rate $\left(\sigma^{2}\right)$ Brownian motion (BM) model (BM1), (2) a BM model with different rate parameters for species having venom gland or not (BMS_gland), (3) a BM model with different rate parameters for species showing a swim bladder or not (BMS_swimbladder), (4) an Ornstein-Uhlenbeck (OU) model with one optimum $(\theta)$ across the entire tree (OU1), (5) an OU model with different optima for species having venom gland (OUM_gland), and (6) an OU model with different optima for species having swimbladder (OUM_swimbladder). We expect that BMS models to best fit our data if morphological innovations mainly influence the rate of size diversification. On the other hand, OUM models should fit better if innovations drive to different size optima.

Modeling of continuous trait evolution was conducted using the OUwie package (Beaulieu et al., 2012) for R, and we compared model fitting using AIC scores and weights. These analyses were ran using 1000 simmap trees, which allow the incorporation of the uncertainty associated with the timing of the transitions between morphological states.

\section{Results}

\subsection{Molecular phylogeny and stochastic mapping}

Our tree topology is generally congruent with previously published studies on the Blenniidae (Hundt et al., 2014; Lin and Hastings 2013). Within the tribe Nemophini, the 15 species of Meiacanthus form a monophyletic group supported by a very long branch that is sister to a clade that includes Aspidontus and Petroscirtes. Constraining the age of crown Blenniidae to 66 Mya (Near et al., 2013), the crown group Meiacanthus dates only to about 6 Mya (95\% CI: 9-4 Mya) (Fig. 1), indicating that the species diversity of this genus arose over a relatively short period of time.

Stochastic mapping revealed that swim bladder appeared once in Nemophini and it was secondarily lost in the genus Xiphasia. A buccal venom gland characterized the common ancestor to all extant Meiacanthus (Fig. 2).

\subsection{Lineage diversification}

The phylorate plot obtained from BAMM analyses showed a relatively constant speciation rate through time among the Blenniidae except for the Meiacanthus clade (Fig. 3A). The post-burn MCMC result revealed that a single rate shift configuration had the highest posterior probability (0.88). We recovered a Bayes factor support for a model with one rate shift of 30.8 over a model with zero rate shifts (Table 1 ). 


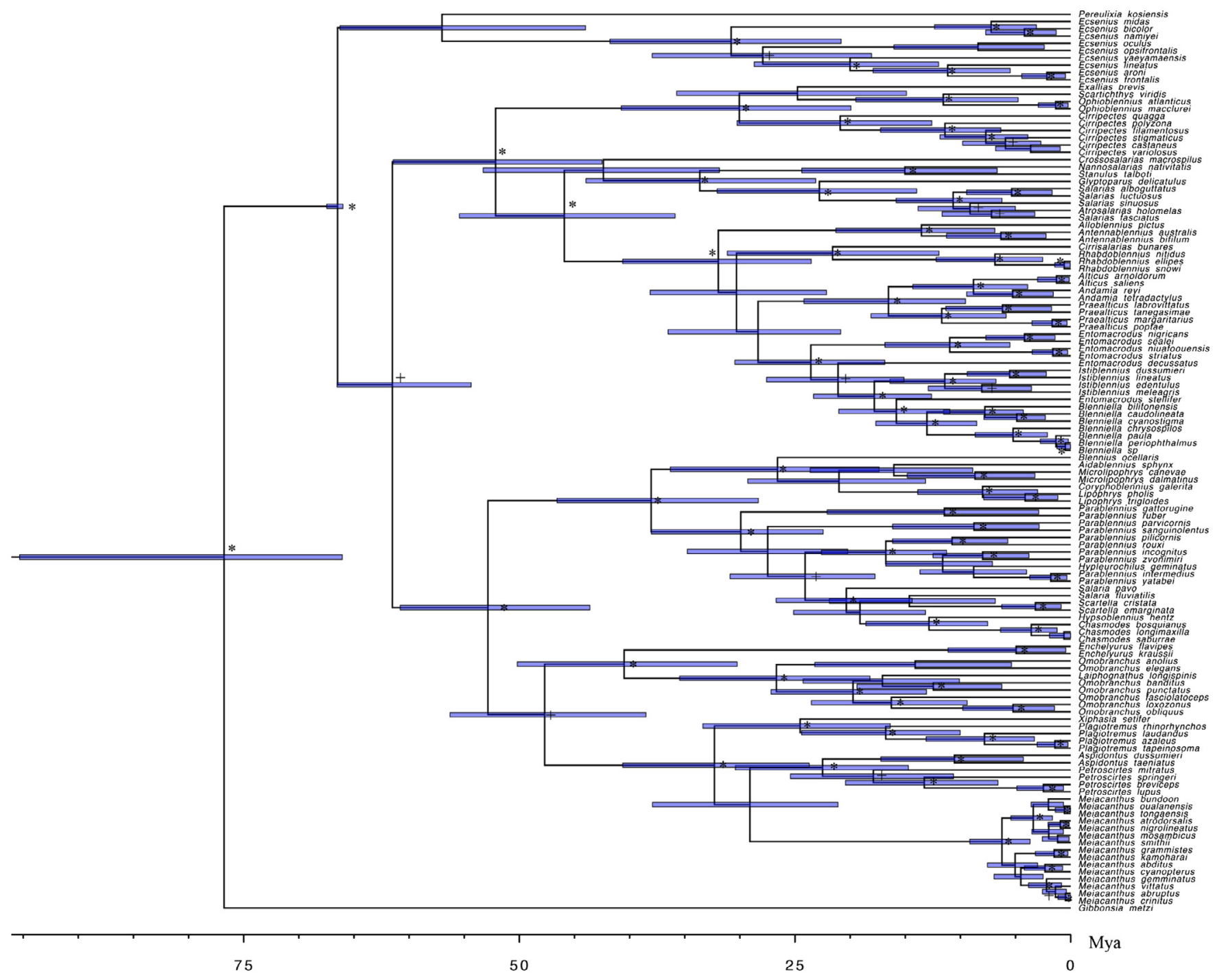

Fig. 1. Time-tree of Blenniidae obtained from Bayesian relaxed clock analysis of the concatenated dataset. Horizontal grey bars at nodes indicate $95 \%$ posterior probability densities (HPD) intervals of age. The horizontal black bar indicates the fossil calibration point (crown age of Blenniidae). * indicates the Bayesian posterior probability of the clade when $>0.95$. + indicates the posterior probability in the range between 0.85 and 0.95 .

This result strongly favored a one-shift model because Bayes factors exceeding 20 are generally considered to be strong evidence in favor of that model. This rate-shift configuration was mapped to the phylogeny of blennies, which showed speciation rate acceleration along with the Meiacanthus clade (Fig. 3A). The credible set of macroevolutionary shift configurations revealed that $99 \%$ of the samples in the posterior can be assigned to a single shift configuration: specifically, the node where the Meiacanthus clade underwent a major increase in speciation rate (Fig. 3B). Macroevolutionary cohort analysis displays the pairwise probability that any two species share a common macroevolutionary rate dynamic. Each cell of the cohort matrix corresponds to a pair of tip taxa from blenny phylogeny-a share rate dynamic between taxa code with warm colors, whereas colder colors indicate that tips do not share a common rate dynamic. Our results showed two general macroevolutionary dynamics across blennies-the Meiacanthus clade and rest of the blenniid lineages. The former represented the "fast clade" and the latter represented "slow lineages" (Fig. 4).

To compare the speciation rate of the Meiacanthus clade to the background speciation rate (Meiacanthus excluded), speciation rates through time were plotted separately. Results show that the speciation rate of Meiacanthus was higher than the background rate, which was elevated rapidly during the past eight million years. On the contrary, the background speciation rate increased very gradually over the past 60 million years (Supplementary Fig. S1).

BiSSE models were used to investigate whether one of the morphological novelties (buccal venom gland and swim bladder) had an effect on the lineage diversification rate of blennies. BiSSE analyses suggested that the presence of a swim bladder did not induce variation in the net diversification rate in Nemophini but lineages with buccal venom glands (Meiacanthus) have a diversification rate four times higher than lineages without venom glands $\left(\lambda_{1}=0.467>\lambda_{0}=0.143\right.$; Supplementary Fig. S2, Tables S2 and S3).

Acknowledging some weakenesses of the BiSSE model (Rabosky and Goldberg, 2015), we also used the HiSSE method (Beaulieu and O'Meara, 2016) in order to explore if a hidden state could contribute to the variation of diversification rates between lineages having a buccal venom gland or not. Our best character-independent diversification model had symmetric transition rates $\left(\mathrm{q}_{01}=\mathrm{q}_{10}\right)$ and no simultaneous transitions between the observed state and the hidden state (Table 2. However, the full BiSSE model was still the best character-independent diversification model according to AIC scores, suggesting that diversification rates were indeed coupled to the presence of a buccal venom gland (Table 4). 


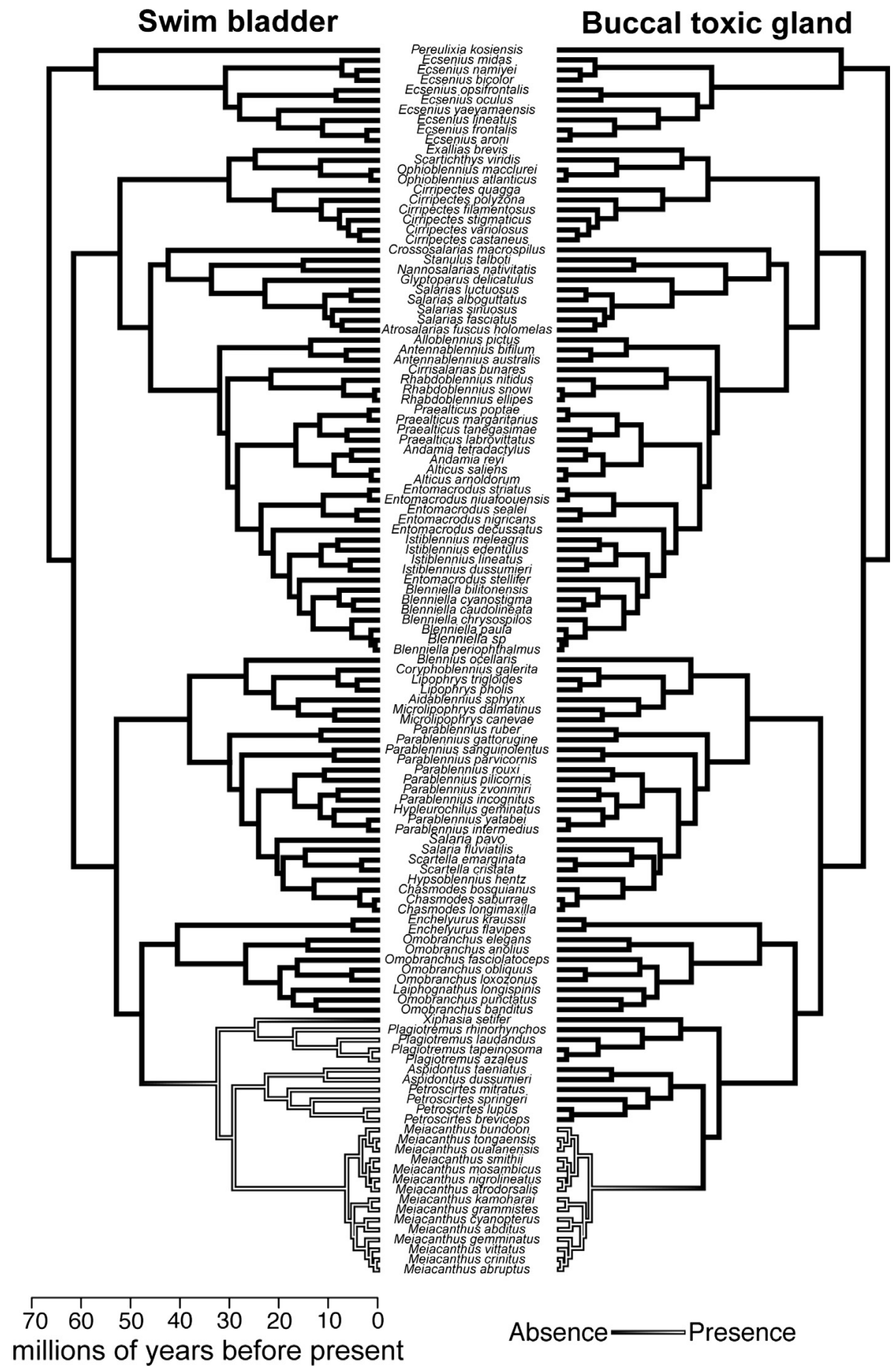

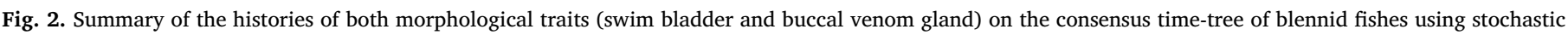
mapping. White refers to the presence of the trait when black depicts its absence.

\subsection{Size disparification}

The effect of each morphological novelty on the pattern of size disparification is limited. Indeed, the null hypothesis that body size in blennies evolved along a unique size optimum value could not be rejected $(\theta=9.3 \mathrm{~cm}$, Table 3$)$.

\section{Discussion}

Our macroevolutionary analyses show that the appearance of buccal venom gland rather than swim bladder has impacted the tempo of diversification in blennies. Indeed, our results revealed a recent burst of speciation at the base of the Meiacanthus clade. This genus is unique in having a buccal venom gland and it has evolved four times faster than other blennies lacking this unique functional trait. On the other hand, neither the appearance of swim bladder nor that of the venom gland affected the pattern of size evolution in Blenniidae.

In marine teleosts, several traits have been proposed as key innovations that play important roles in the diversification of several taxonomic groups, examples including antifreeze glycoproteins (AFGPs) in Antarctic notothenioid fishes (Near et al., 2012) and pharyngeal jaw apparatus (PJA) in labrid fishes (Alfaro et al., 2009). 
A

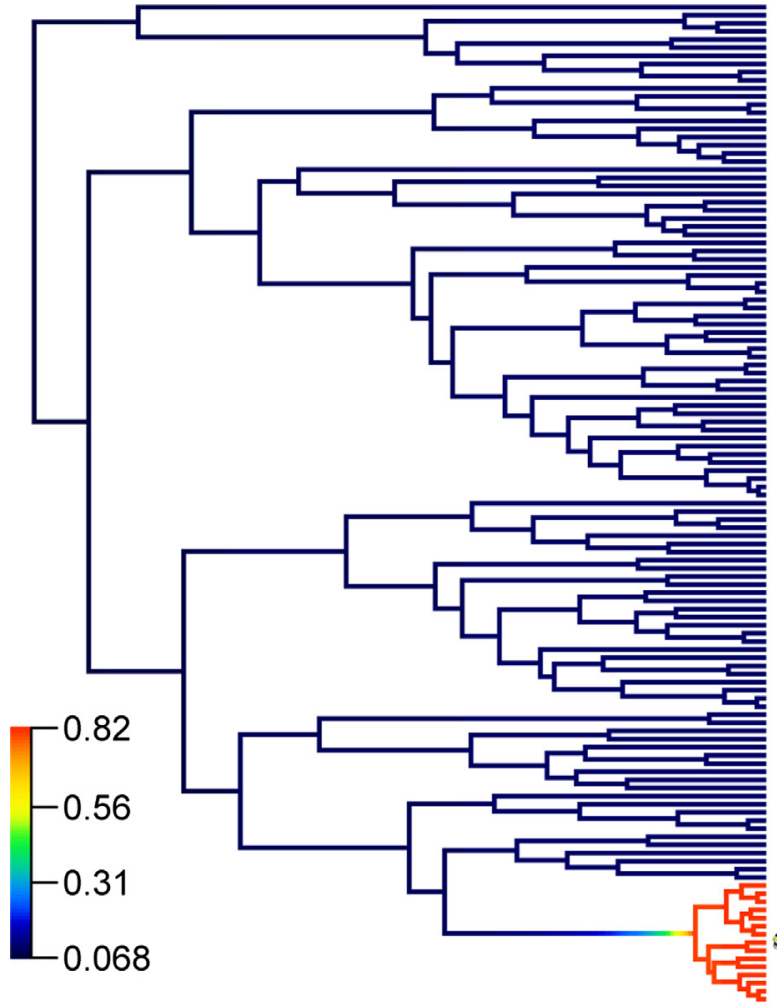

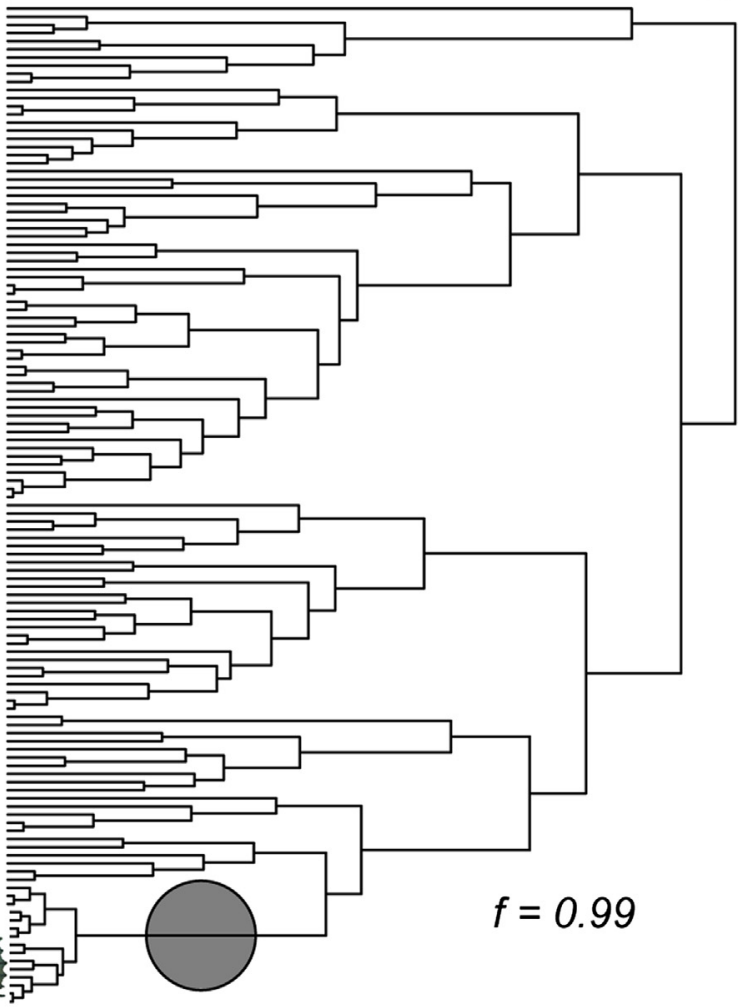

Fig. 3. (A) Bayesian phylogeny of blennies, with BAMM estimates of instantaneous speciation rate represented by colors along individual branches and (B) the $95 \%$ credible set of macroevolutionary shift configurations.

Table 1

Bayes factor support for diversification rate shifts.

\begin{tabular}{lll}
\hline & Bayes factor & Interpretation \\
\hline 1 vs. 0 shifts & 30.87 & Strong support for 1 shift over 0 shifts \\
1 vs. 2 shifts & 43.02 & Very strong support for 1 shift over 2 shifts \\
0 vs. 2 shifts & 1.39 & Barely any support for 0 shifts over 2 shifts \\
\hline
\end{tabular}

However, it have been observed that species diversification within these taxa is not always synchronized to the evolution of the proposed key innovation (Wainwright et al., 2012). For instance, the origin of AFGPs and PJA substantially predated the diversification event observed in the time-calibrated trees of notothenioids and labrids, respectively (Alfaro et al., 2009; Near et al., 2012). This pattern implies that getting a new trait may not immediately spur lineage diversification because ecological opportunity and fitness gain only when they explore new niches (Alfaro et al., 2009; Near et al., 2012). Such examples demonstrate the difficulty in linking the key innovation hypotheses to key traits that trigger diversification. In the present study, the results of BAMM strongly support a single rate-shift linked to the long branch of the Meiacanthus clade. Additionally, results from BiSSE and HiSSE models showed that these lineages with buccal venom glands have a higher speciation rate than lineages without this trait. In addition to the buccal venom gland, the presence of the swim bladder at the adult stage may also impact evolutionary patterns within the Blenniidae. The Nemophini, unlike their close relatives, are semi-pelagic active swimmers facilitated by a well-developed swim bladder. Its ecological function is not well-studied in blennies but it has been suggested that it facilitates the formation of mimicking behaviors in the Nemophini (Smith-Vaniz and Allen, 2011; Robertson, 2013). However, unlike the buccal venom gland, the BiSSE and HiSSE results showed that there is no sign of increasing diversification rate due to swim bladder in the Nemophini, except the primary influence by this trait on the diversification of Meiacanthus. Consequently we cannot exclude the possible interaction between the buccal venom gland and swim bladder on the burst of lineage diversification since they are both present in Meiacanthus. Their success is very likely linked to the combination of both novelties, allowing them to diversify along various previously unexplored ecological niches.

Body size is an important feature of any animal. It determines potential predators and prey (Peters, 1983). Size diversification may also be strongly associated with ecological differentiation, which allows animals to access available habitats or unused resources (Bernatchez et al., 1999; Peters, 1983; Polo and Carrascal, 1999). Here, the effect of both morphological novelties on the pattern of size evolution in Blenniidae is limited. Speciation is not associated with size variation among Meiacanthus species. However we cannot reject the hypothesis that the observed higher rate of speciation in Meiacanthus is linked to the invasion of untapped niches previously inaccessible without the studied morphological novelties. For example, the great majority of Meiacanthus species are mainly associated with reef environment but $M$. anema invades freshwater habitats and two species (M. cyanopterus and M. erdmanni) live in deeper reefs ( $>40 \mathrm{M}$ ) (Allen and Erdmann, 2012). We suggest that further ecomorphological, stable isotopes and stomach content analyses are needed to reveal a possible interspecific habitat and trophic partitioning linking lineage and niche differentiation within Meiacanthus species. Moreover, as observed in the venomous cone snails (Phuong et al., 2016), the taxonomic diversification of Meiacanthus could also be associated with inter-specific variation in venom composition and its complexity. Finally, beside ecological and biochemical explanations on the burst of speciation in Meiacanthus, sexual selection may have also played a role in their diversification as observed in other reef fishes (e.g. Kazancioglu et al., 2009).

Species in the genus Meiacanthus are distributed exclusively in the Indo-Pacific, especially in the Indo-Australian Archipelago (IAA), which has very high rates of endemism (Allen and Erdmann, 2012; Smith- 


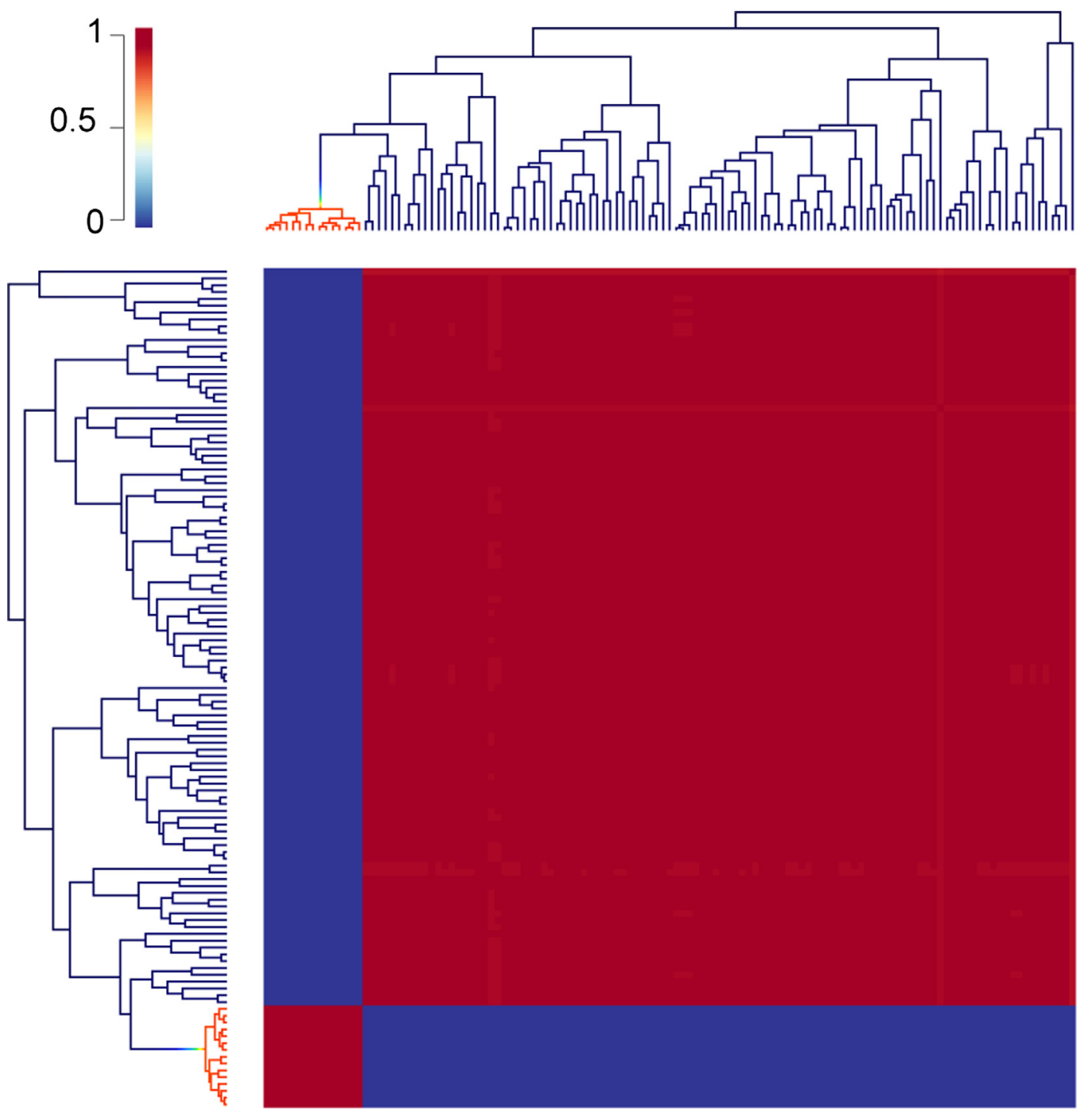

Fig. 4. Macroevolutionary cohort matrix for the MCC phylogeny of blennies. The clade marked with red color is the genus Meiacanthus. There is a strong evidence for Meiacanthus being part of a decoupled diversification regime from other blennies.

Table 2

Character-independent diversification (CID) analysis using HiSSE, AIC: Akaike score; $\lambda$ and $\mu$, speciation and extinction rates; present (1) and absent (0).

\begin{tabular}{|c|c|c|c|c|c|c|}
\hline Model & AIC & $\triangle \mathrm{AIC}$ & $\lambda_{0}$ & $\lambda_{1}$ & $\mu_{0}$ & $\mu_{1}$ \\
\hline BiSSE & 906.8818 & 0.0000 & 0.1508 & 0.431 & 0.0781 & 0 \\
\hline $\begin{array}{c}\text { CID, symmetric } \\
\text { transitions }\end{array}$ & 911.4568 & 4.5750 & 0.7577 & 0.1506 & 0.0143 & 0.0778 \\
\hline BiSSE null & 913.5270 & 6.6452 & 0.1952 & 0.1952 & 0.1293 & 0.1293 \\
\hline $\mathrm{CID}$, all rates equal & 917.6670 & 10.7852 & 0.0058 & 0.1968 & 0.1305 & 0 \\
\hline CID, all rates different & 920.9941 & 14.1123 & 0.1508 & 0.4313 & 0.0781 & 0 \\
\hline
\end{tabular}

Vaniz, 1976, 1987). The IAA is proposed as the center for lineage export/expansion over an extended period (Pliocene - Recent) of time based on the macroevolutionary analyses of three diverse reef fish families (Labridae, Pomacentridae, and Chaetodontidae) (Cowman and Bellwood, 2013). The quaternary period (2.6 million years ago to present) is characterized by at least 30 glacial-interglacial cycles of repeated global cooling and warming, with consequences for reef habitat availability. In the Indo-Pacific, extensive coral reef refugia were preserved and helped nurse the current reef fish biodiversity of IAA as the sources for future recolonization during cold periods (Pellissier et al., 2014). While allopatric divergence in the Indo-Pacific is well
Table 3

Results from fitting size disparification models. The models are ranked from best to worst, according to AICc (small-sample corrected AIC) scores and Akaike weights (wtAIC). $\triangle$ AIC scores indicate the difference between the candidate model and the best-fitting model. Refer to text for model description.

\begin{tabular}{llll}
\hline Model & AICc & AAIC & wtAIC \\
\hline OU1 & -50.43 & 0 & 0.46 \\
OUM_gland & -50.02 & 0.40 & 0.38 \\
OUM_bladder & -48.37 & 2.06 & 0.16 \\
BMS_gland & -35.09 & 15.34 & $2.15 \times 10^{-04}$ \\
BMS_bladder & -24.76 & 25.67 & $1.23 \times 10^{-06}$ \\
BM1 & 7.11 & 57.54 & $1.47 \times 10^{-13}$ \\
\hline
\end{tabular}

documented (Barber et al., 2011; Carpenter et al., 2011), so too is population expansion (Crandall et al., 2011). Sea level fluctuations are implicated in the population expansion and speciation of teleost groups such as damselfishes (Sorenson et al., 2014a, 2014b), and have been suggested to associate with the origins and rapid radiation of grouper lineages (Ma et al., 2016). Within Meiacanthus, as the shallow coastal regions of the Indo West Pacific were resubmerged following glacial retreat, fang blennies would likely have had unique opportunities to expand their geographic distribution into newly available habitats, 
providing opportunities to adapt to new environments and potentially explaining the explosive diversification in Meiacanthus reported in this study. Colonization into newly available habitats in the coastal regions of the Indo-Pacific may also explain the largely restricted range of Meiacanthus, with the exception of $M$. atrodorsalis, M. ditrema, $M$. grammistes, and M. anema (Allen and Erdmann, 2012; Smith-Vaniz, 1976, 1987).

\section{Conclusion}

To conclude, we show that the evolution of the buccal venom gland or its correlated evolution with the appearance of a swim bladder resulted in an elevated speciation rate within Meiacanthus. The function of these traits may have helped to expand their habitats following Pliocene glacial cycles. There are many teleost groups with their center of diversity in the Indo-Pacific. New analytical tools such as those employed in this study may provide new evidences highlighting periods of increased speciation rates linked to the appearance of novel morphologies or behaviors that may have spurred this diversification.

\section{Acknowledgements}

We would like to thank Andrew Bentley (Kansas University) and H.J. Walker (Scripps institute of oceanography) for curatorial help and the staffs of IBRC (Indonesia Biodiversity Research Center) for logistic and field support. Research activities and sample collections in Indonesia were under research permit No. 272/SIP/FRP/SM/VII/2013. This research was supported by a postdoctoral fellowship (NSC 1022917-I-564-015) and a grant issued by the Ministry of Science and Technology of Taiwan (104-2611-M-110-022-MY2).

\section{Appendix A. Supplementary material}

Supplementary data associated with this article can be found, in the online version, at http://dx.doi.org/10.1016/j.ympev.2018.03.027.

\section{References}

Alfaro, M.E., Santini, F., Brock, C.D., 2007. Do reefs drive diversification in marine teleosts? Evidence from the pufferfish and their allies (order Tetraodontiformes). Evolution 61, 2104-2126.

Alfaro, M.E., Brock, C.D., Banbury, B.L., Wainwright, P.C., 2009. Does evolutionary in novation in pharyngeal jaws lead to rapid lineage diversification in labrid fishes? BMC Evol. Biol. 22, 255.

Allen, G.R., Erdmann, M.V., 2012. Reef fishes of the East Indies. Tropical Reef Research, Perth.

Bannikov, A.F., 1998. New blennioid fishes of the families Blenniidae and Clinidae (Perciformes) from the Miocene of the Caucasus and Moldova. Paleontol. J. 32, 385-389.

Bannikov, A.F., 2014. The systematic composition of the Eocene actinopterygian fish fauna from Monte Bolca, northern Italy, as known to date. Studi e Ricerche sui Giacimenti Terziari di Bolca 15, 23-34.

Barber, P.H., Cheng, S.H., Erdmann, M.V., Tengardjaja, K., Ambariyanto, A., 2011 Phylogeography and population genetics in Crustacea: evolution and conservation of marine biodiversity. In: Held, C., Koenemann, S., Schubart, C.D. (Eds.), The Coral Triangle: Insights from Stomatopod Crustacea. CRC Press, pp. 129-156.

Beaulieu, J.M., Jhwueng, D.C., Boettiger, C., O'Meara, B.C., 2012. Modeling stabilizing selection: expanding the Ornstein-Uhlenbeck model of adaptive evolution. Evolution $66,2369-2383$

Beaulieu, J.M., O'Meara, B.C., 2016. Detecting hidden diversification shifts in models of trait-dependent speciation and extinction. Syst. Biol. 65, 583-601.

Bernatchez, L., Chouinard, A., Lu, G., 1999. Integrating molecular genetics and ecology in studies of adaptive radiation: whitefish, Coregonus sp., as a case study. Biol. J. Linn. Soc. 68, 173-194.

Betancur, R.R., Ortí, G., Stein, A.M., Marceniuk, A.P., Alexander, P.R., 2012. Apparent signal of competition limiting diversification after ecological transitions from marine to freshwater habitats. Ecol. Lett. 15, 822-830.

Carlson, B.A., Hasan, S.M., Hollmann, M., Miller, D.B., Harmon, L.J., Arnegard, M.E., 2011. Brain evolution triggers increased diversification of electric fishes. Science 332, $583-586$.

Carpenter, K.E., Barber, P.H., Crandall, E.D., Ablan-Lagman, M., Carmen, A., Mahardika, G.N., Manjaji-Matsumoto, B.M., Juinio-Meñez, M.A., Santos, M.D., Starger, C.J., Toha, A.H.A., 2011. Comparative phylogeography of the Coral Triangle and implications for marine management. J. Mar. Biol. (ID 396982)
Casewell, N.R., Visser, J.C., Baumann, K., Dobson, J., Han, H., Kuruppu, S., Morgan, M., Romilio, A., Weisbecker, V., Mardon, K., Ali, S.A., Debono, J., Koludarov, I., Que, I., Bird, G.C., Cooke, G.M., Nouwens, A., Hodgson, W.C., Wagstaff, S.C., Cheney, K.L., Vetter, I., van der Weerd, L., Richardson, M.K., Fry, B.G., 2017. The evolution of fangs, venom, and mimicry systems in blenny fishes. Curr. Biol. 27, 1184-1191.

Cowman, P.F., Bellwood, D.R., 2013. The historical biogeography of coral reef fishes: global patterns of origination and dispersal. J. Biogeogr. 40, 209-224.

Crandall, E.D., Sbrocco, E.J., DeBoer, T.S., Barber, P.H., Carpenter, K.E., 2011. Expansion dating: calibrating molecular clocks in marine species from expansions onto the Sunda Shelf following the Last Glacial Maximum. Mol. Biol. Evol msr227.

Darriba, D., Taboada, G.L., Doallo, R., Posada, D., 2012. jModelTest 2: more models, new heuristics and parallel computing. Nat. Methods 9, 772

Drummond, A.J., Rambaut, A., 2007. BEAST: Bayesian evolutionary analysis by sampling trees. BMC Evol. Biol. 7, 214.

Fishelson, L., 1974. Histology and ultrastructure of the recently found buccal toxic gland in the fish Meiacanthus nigrolineatus (Blenniidae). Copeia 1974, 386-392.

FitzJohn, R.G., 2012. Diversitree: comparative phylogenetic analyses of diversification in R. Methods Ecol. Evol. 3, 1084-1092.

Frédérich, B., Marrama, G., Carnevale, G., Santini, F., 2016. Non-reef environments impact the diversification of extant jacks, remoras and allies (Carangoidei, Percomorpha). Proc. R. Soc. B 283, 20161556.

Frédérich, B., Olivier, D., Litsios, G., Alfaro, M.E., Parmentier, E., 2014. Trait decoupling promotes evolutionary diversification of the trophic and acoustic system of damselfishes. Proc. R. Soc. B 281, 20141047.

Fry, B.G., Vidal, N., Norman, J.A., Vonk, F.J., Scheib, H., Ramjan, S.F., Kuruppu, S., Fung, K., Hedges, S.B., Richardson, M.K., Hodgson, W.C., Ignjatovic, V., Summerhayes, R., Kochva, E., 2006. Early evolution of the venom system in lizards and snakes. Nature 439, 584-588.

Hastings, P.A., Springer, V.G., 2009. Systematics of the Blenniidae (combtooth blennies), In: Patzer, R.A., Goncalves, E.J., Hastings, P.A., Kapoor, B.G. (Eds.), The Biology of Blennies. CRC Press, pp. 3-30.

Heard, S.B., Hauser, D.L., 1995. Key evolutionary innovations and their ecological mechanisms. Hist. Biol. 10, 151-173.

Helmstetter, A.J., Papadopulos, A.S., Igea, J., Van Dooren, T.J., Leroi, A.M., Savolainen, V., 2016. Viviparity stimulates diversification in an order of fish. Nat. Commun. 7, 11271.

Hundt, P.J., Iglésias, S.P., Hoey, A.S., Simons, A.M.A., 2014. A multilocus molecular phylogeny of combtooth blennies (Percomorpha: Blennioidei: Blenniidae): Multiple invasions of intertidal habitats. Mol. Phyl. Evol 70, 47-56.

Kazancioglu, E., Near, T.J., Hanel, R., Wainwright, P.C., 2009. Influence of sexual selection and feeding functional morphology on diversification rate of parrotfishes (Scaridae). Proc. R. Soc. B 276, 3439-3446.

Levinton, J.S., 1988. Genetics, Paleontology, and Macroevolution. Cambridge University Press, United Kingdom.

Li, C., Ortí, G., Zhang, G., Lu, G.A., 2007. practical approach to phylogenomics: the phylogeny of ray-finned fish (Actinopterygii) as a case study. BMC Evol. Biol. 7, 1.

Lin, H.C., Hastings, P.A., 2013. Phylogeny and biogeography of a shallow water fish clade (Teleostei: Blenniiformes). BMC Evol. Biol. 13, 1.

Litsios, G., Sims, C.A., Wüest, R.O., Pearman, P.B., Zimmermann, N.E., Salamin, N., 2012 Mutualism with sea anemones triggered the adaptive radiation of clownfishes. BMC Evol. Biol. 12, 1.

Losos, J.B., 2010. Adaptive radiation, ecological opportunity, and evolutionary determinism. Am. Nat. 175, 623-639.

Ma, K.Y., Craig, M.T., Choat, J.H., van Herwerden, L., 2016. The historical biogeography of groupers: clade diversification patterns and processes. Mol. Phyl. Evol. 100, 21-30.

Maddison, W.P., Midford, P.E., Otto, S.P., 2007. Estimating a binary character's effect on speciation and extinction. Syst. Biol. 56, 701-710.

Near, T.J., Dornburg, A., Eytan, R.I., Keck, B.P., Smith, W.L., Kuhn, K.L., Moore, J.A., Price, S.A., Burbrink, F.T., Friedman, M., Wainwright, P.C., 2013. Phylogeny and tempo of diversification in the superradiation of spiny-rayed fishes. Proc. Natl. Acad. Sci. U. S. A. 101, 12738-21743.

Near, T.J., Dornburg, A., Kuhn, K.L., Eastman, J.T., Pennington, J.N., Patarnello, T., Zane, L., Fernández, D.A., Christopher, D.J., 2012. Ancient climate change, antifreeze, and the evolutionary diversification of Antarctic fishes. Proc. Natl. Acad. Sci. U. S. A. 109 3434-3439.

Olivier, D., Gajdzik, L., Parmentier, E., Frédérich, B., 2017. Evolution and diversity of ram-suction feeding in damselfishes (Pomacentridae). Organ. Divers. Evol. 17 497-508.

Pellissier, L., Leprieur, F., Parravicini, V., Cowman, P.F., Kulbicki, M., Litsios, G., Olsen, S.M., Wisz, M.S., Bellwood, D.R., Mouillot, D., 2014. Quaternary coral reef refugia preserved fish diversity. Science 344, 1016-1019.

Peters, R.H., 1983. The Ecological Implications of Body Size. Cambridge University Press, Cambridge.

Phuong, M.A., Mahardika, G.N., Alfaro, M.E., 2016. Dietary breadth is positively correlated with venom complexity in cone snails. BMC Genom. 17, 401.

Plummer, M., Best, N., Cowles, K., Vines, K., 2006. CODA: convergence diagnosis and output analysis for MCMC. R News 6, 7-11.

Polo, V., Carrascal, L.M., 1999. Shaping the body mass distribution of Passeriformes: habitat use and body mass are evolutionarily and ecologically related. J. Anim. Ecol. 68, 324-337.

Price, S.A., Tavera, J., Near, T.J., Wainwright, P.C., 2013. Elevated rates of morphological and functional diversification in reef-dwelling haemulid fishes. Evolution 67, $417-428$.

Santini, F., Nguyen, M.T.T., Sorenson, L., Waltzek, T.B., Alfaro, J.W.L., Eastman, J.M., Alfaro, M.E., 2013. Do habitat shifts drive diversification in teleost fishes? An example from the pufferfishes (Tetraodontidae). J. Evol. Biol. 26, 1003-1018. 
Shaun, K.W., 2009. Diversity in the diet and feeding habitats of blennies. In: Patzer, R.A., Goncalves, E.J., Hastings, P.A., Kapoor, B.G. (Eds.), The Biology of Blennies. CRC Press, pp. 69-91.

Shi, J.J., Rabosky, D.L., 2015. Speciation dynamics during the global radiation of extant bats. Evolution 69, 1528-1545.

Simpson, G.G., 1953. The Major Features of Evolution. Columbia University Press, New York.

Smith, W.L., Wheeler, W.C., 2006. Venom evolution widespread in fishes: a phylogenetic road map for the bioprospecting of piscine venoms. J. Hered. 97, 206-217.

Smith-Vaniz, W.F., 1976. Saber-Toothed Blennies, Tribe Nemophini (Pisces: Blenniidae). Acad. Nat. Sci. Phila. Monogr.

Smith-Vaniz, W.F., 1987. The saber-toothed blennies, tribe Nemophini (Pisces: Blenniidae): an update. Proc. Acad. Nat. Sci. Phila. 139, 1-52.

Smith-Vaniz, W.F., 2017. A new species of the fangblenny Adelotremus from Indonesia, with supplemental description of A. leptus (Teleostei: Blenniidae: Nemophini). Zootaxa 4258, 179-186.

Smith-Vaniz, W.F., Allen, G.R., 2011. Three new species of the fangblenny genus Meiacanthus from Indonesia, with color photographs and comments on other species (Teleostei: Blenniidae: Nemophini). Zootaxa 3046, 39-58.

Smith-Vaniz, W.F., Satapoomin, U., Allen, G.R., 2001. Meiacanthus urostigma, a new fangblenny from the northeastern Indian Ocean, with discussion and examples of mimicry in species of Meiacanthus (Teleostei: Blenniidae: Nemophini). Aqua J. Ichthyol. Aquat. Biol. 5, 25-43.

Sorenson, L., Allen, G.R., Erdmann, M.V., Dai, C.F., Liu, S.Y.V., 2014a. Pleistocene diversification of the Pomacentrus coelestis species complex (Pisces: Pomacentridae): historical biogeography and species boundaries. Mar. Biol. 161, 2495-2507.

Sorenson, L., Santini, F., Alfaro, M.E., 2014b. The effect of habitat on modern shark diversification. J. Evol. Biol. 27, 1536-1548.

Rabosky, D.L., Donnellan, S.C., Grundler, M., Lovette, I.J., 2014. Analysis and visualization of complex macroevolutionary dynamics: an example from Australian scincid lizards. Syst. Biol. 63, 610-627.

Rabosky, D.L., Goldberg, E.E., 2015. Model inadequacy and mistaken inferences of traitdependent speciation. Syst. Biol. 64, 340-355.

R Core Team, 2013. R: A Language and Environment for Statistical Computing. R Foundation for Statistical Computing, Vienna, Austria.

Revell, L.J., 2012. Phytools: an R package for phylogenetic comparative biology (and other things). Methods Ecol. Evol. 3, 217-223.

Robertson, D.R., 2013. Who resembles whom? Mimetic and coincidental look-alikes among tropical reef fishes. Plos One 8, e54939.

Vidal, N., 2002. Colubroid systematics: evidence for an early appearance of the venom apparatus followed by extensive evolutionary tinkering. J. Toxicol.: Toxin. Rev. 21, $21-41$.

Wainwright, P.C., Price, S.A., 2016. The impact of organismal innovation on functional and ecological diversification. Integr. Comp. Biol. 56, 479-488.

Wainwright, P.C., Smith, W.L., Price, S.A., Tang, K.L., Sparks, J.S., Ferry, L.A., Kuhn, K.L., Eytan, R.I., Near, T.J., 2012. The evolution of pharyngognathy: a phylogenetic and functional appraisal of the pharyngeal jaw key innovation in labroid fishes and beyond. Syst. Biol. 61, 1001-1027.

Yabumoto, Y., Uyeno, T., 2007. Tottoriblennius hiraoi, a new genus and species of Miocene blennioid fish from Tottori Prefecture, Japan. Bull. Natl. Mus. Natl. Sci., C 33, 81-87. 